Notfall Rettungsmed 2017 $\cdot 20: 161-162$

DOI 10.1007/s10049-016-0217-y

Online publiziert: 26. August 2016

(c) Die Autoren 2016. Dieser Artikel ist eine

Open-Access-Publikation.

CrossMark

\section{Anamnese}

An einem Herbstabend wurde die berichtende Mannschaft eines Rettungstransportwagens zu einem 47-jährigen Mann mit Thoraxschmerz berufen. In der Wohnung fand sich ein deutlich übergewichtiger, blasser und stark schwitzender Mann, der die geballte Faust an die Brust hielt.

Es bestanden seit etwa $1 \mathrm{~h}$ anhaltende, drückend-brennende Thoraxschmerzen, die in den linken Arm ausstrahlten und vom Patienten mit einer Stärke von 8/10 beschrieben wurden. Die Schmerzen seien in Ruhe aufgetreten und nicht atemoder bewegungsabhängig.

Der Patient wurde nach dem ABCDESchema untersucht: Der Atemweg war frei, keine Dyspnoe, $\mathrm{S}_{\mathrm{p}} \mathrm{O}_{2} 100 \%$. Auskultatorisch bestand beidseits ein vesikuläres Atemgeräusch ohne Rasselgeräusche; die Herztöne waren leise, jedoch rein und rhythmisch.

Die Pulse waren überall gut tastbar, der Blutdruck betrug beidseits 205/126 mm Hg. Das EKG zeigte einen Sinusrhythmus mit einer Frequenz von $89 / \mathrm{min}$, die ST-Strecken waren isoelektrisch (- Abb. 1).

Neurologisch war der Patient unauffällig. Es bestand eine Erdnussallergie; Dauermedikation: $5 \mathrm{mg}$ Enalapril wegen Hypertonie. Familienanamnestisch waren mehrere Herzinfarkte und Insulte erhebbar.

\section{Therapie und Verlauf}

Unter der Arbeitsdiagnose „hypertensive Entgleisung mit AP-Symptomatik“ wurde die Indikation zur akuten Senkung des Blutdrucks gestellt. Nach Aufklärung des Patienten wurde eine 18-G-Venen-

\author{
M. Müller ${ }^{1,2} \cdot$ M. Koch ${ }^{1,2}$ \\ ${ }^{1}$ Medizinische Universität Wien, Wien, Österreich \\ ${ }^{2}$ Wiener Rotes Kreuz Rettungsdienst, Wien, Österreich
}

\title{
Bradykardie nach Gabe von Nitrospray
}

verweilkanüle gelegt und gemäß des örtlichen Algorithmus zur Therapie des vorliegenden Krankheitsbildes ein Hub Nitroglycerin 0,4 mg sublingual verabreicht. Nach 6 min betrug der Blutdruck immer noch 195/120 mm Hg, die Beschwerden waren unverändert. Abermals wurde ein Hub Nitroglycerin gegeben, woraufhin sich der Zustand des Patienten dramatisch veränderte: Er klagte über Schwindel, war fahl im Gesicht, präkollaptisch und kaltschweißig. Die Herzfrequenz fiel auf zeitweise unter 30/min ab und blieb bei durchgehender Sinusbradykardie für etwa 1,5 min auf solchen Werten (• Abb. 2).

Der erneut gemessene Blutdruck betrug 86/40 mm Hg, sodass der Patient schockgelagert und die rasche Infusion einer Vollelektrolytlösung begonnen wurde. Daraufhin besserte sich der Patientenzustand rasch, Frequenz und Blutdruck stiegen wieder. Durch den nachgeforderten Notarzt erfolgte keine weitere Therapie. Im Krankenhaus kam es in seriellen Kontrollen (23:00 h, 02:00 h) zu keiner Auslenkung des Troponins; auch das D-Dimer blieb negativ. Der Patient wurde in derselben Nacht beschwerdefrei entlassen und eine weiterführende Abklärung beim niedergelassenen Internisten empfohlen.

\section{Diskussion}

Plötzlicher Herzfrequenzabfall nach Nitroglyceringabe ist eine eher ungewöhnliche Nebenwirkung, auch wenn sie in der Literatur beschrieben ist [5]. Als mögliche Pathomechanismen wurden der Bezold-Jarisch-Reflex (BJR) sowie eine zentrale Sympathikusinhibition im Nucleus tractus solitarii beschrieben.
Der BJR ist ein pathophysiologisch nicht abschließend geklärter kardioinhibitorischer Reflex: Niedrige Füllungsdrücke im linken Ventrikel führen $\mathrm{zu}$ verstärkter Kontraktion des Herzens. Es kommt zur Aktivierung intrakardialer Presso- und Chemorezeptoren, die wiederum zu vagaler Stimulation führen und so Hypotension auslösen [4]. Der BJR dürfte außerdem den Barorezeptorreflex inhibieren, sodass auf die Hypotension keine Reflextachykardie, sondern Bradykardie folgt [6]. Im konkreten Fall sind als begünstigende Faktoren zur Auslösung eines BJR weitere klinische Bedingungen in Betracht zu ziehen: ein durch die vermutlich länger anhaltende Hypertension bestehender Volumenmangel und möglicherweise eine linksventrikuläre Hypertrophie. Beide können die rasche Entstehung einer nicht mehr ausreichenden Füllung im linken Ventrikel bedingen. Der zügige Wirkungseintritt von $\mathrm{Ni}$ troglycerin mit Vasodilatation trägt zusätzlich dazu bei. Als weitere, den BJR auslösende Differenzialdiagnose ist eine „small vessel disease“ zu nennen. Hierbei kommt es auf Basis einer (in diesem Fall am ehesten hypertensiven) Mikroangiopathie der kleinen intramuralen Koronargefäße zu pektanginösen Beschwerden, ohne dass in den großen epikardialen Gefäßen Stenosen nachweisbar sind. Es gibt Berichte von Patienten ohne angiographisch nachweisbare Stenosen, die im Rahmen einer Koronarangiographie einen BJR entwickelten. Esente et al. vermuten als Ursache dafür am ehesten Veränderungen des myokardialen Blutflusses durch Nitroglycerin oder Kontrastmittel [1].

Eine zweite Theorie zur Ursache der Bradykardie ist eine direkte, zentral- 


\section{Der interessante Fall}

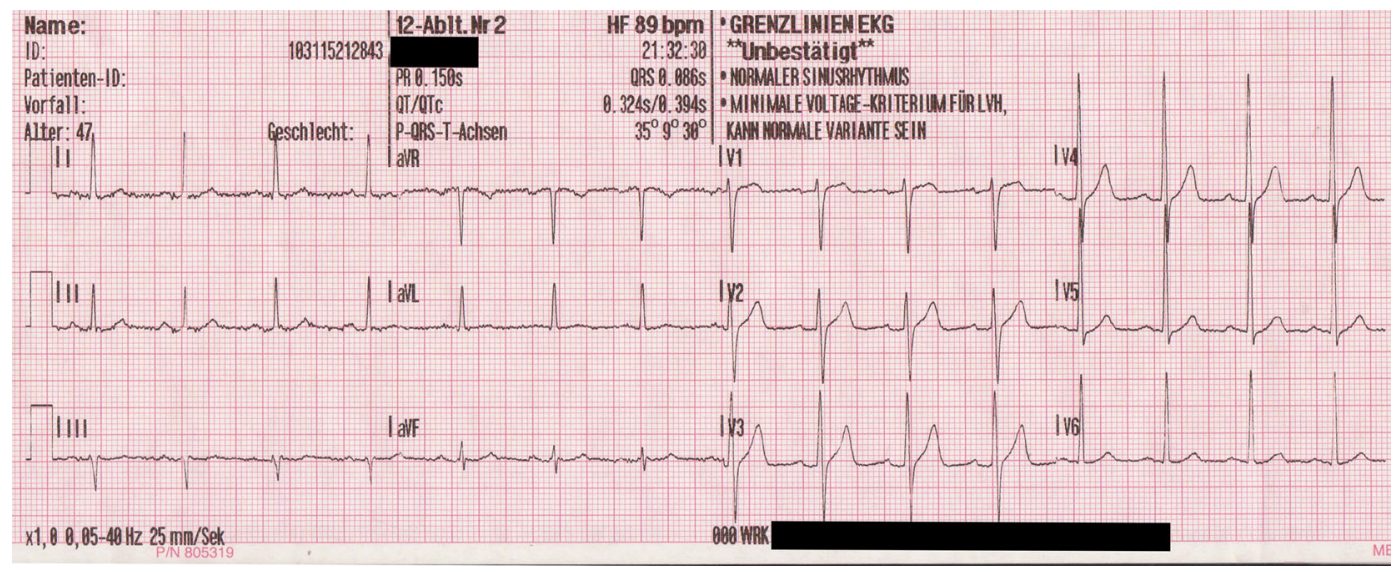

Abb. $1 \varangle$ Sinusrhythmus, 89/min, Linkstyp, unauffällige ST-Strecken, grenzwertige Hypertrophiezeichen

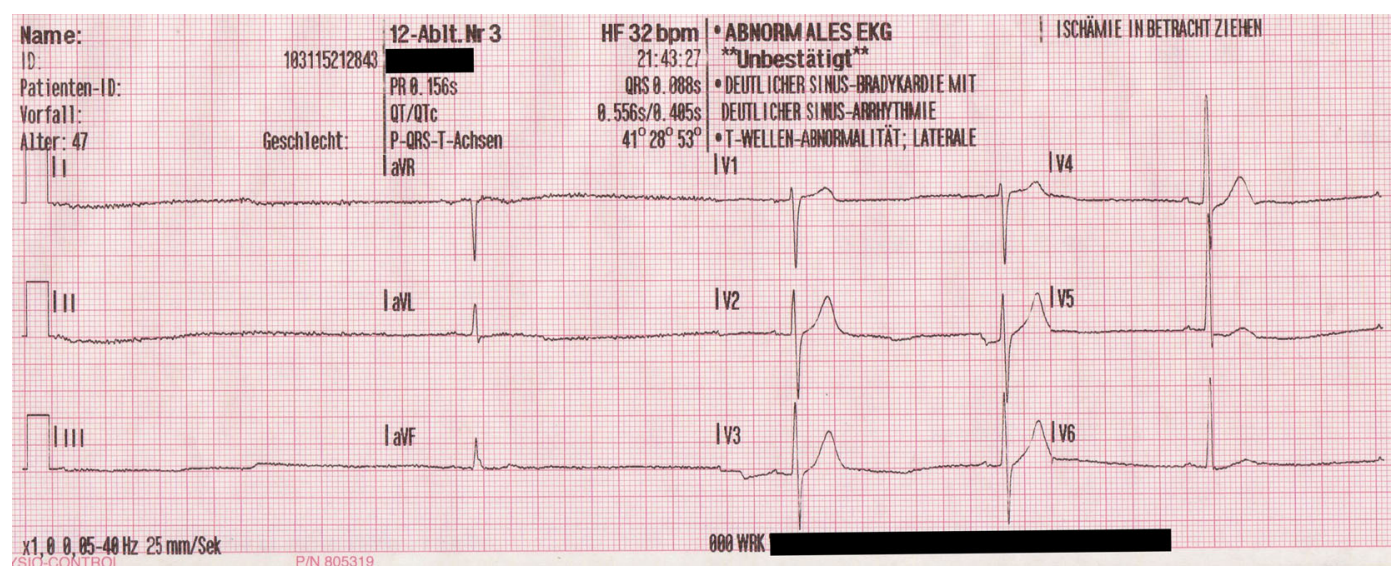

Abb. $2<$ Sinusbradykardie mit Frequenz $<30 / \mathrm{min}$

nervöse Wirkung des Medikaments: An Ratten konnte gezeigt werden, dass es durch Nitroglycerininjektion in den Nucleus tractus solitarii (NTS) zu einer zentralen Sympathikusblockade mit Bradykardie und Hypotension kommt $[2,3]$. Eine direkte Wirkung im NTS ist aufgrund der hohen Lipophilie von Nitroglycerin durchaus denkbar.

\section{Fazit für die Praxis}

Auch wenn eine durch Nitroglycerin verursachte Bradykardie eine eher seltene Nebenwirkung darstellt, kann dieser Effekt im medizinischen Alltag durchaus vorkommen. Es gilt, auf einen solchen Zwischenfall vorbereitet zu sein.

\section{Korrespondenzadresse}

\section{Müller}

Wiener Rotes Kreuz Rettungsdienst Nottendorfer Gasse 21, 1030 Wien, Österreich matthias.mueller@meduniwien.ac.at

Open access funding provided by Medical University of Vienna.

\section{Einhaltung ethischer Richtlinien}

Interessenkonflikt. M. Müller und M. Koch geben an, dass kein Interessenkonflikt besteht.

Angaben zum Patienten wurden soweit anonymisiert, dass ein Erkennen des Patienten nicht möglich sein sollte.

Open Access. Dieser Artikel wird unter der Creative Commons Namensnennung 4.0 International Lizenz (http://creativecommons.org/licenses/by/4.0/deed. de) veröffentlicht, welche die Nutzung, Vervielfältigung, Bearbeitung, Verbreitung und Wiedergabe in jeglichem Medium und Format erlaubt, sofern
Sie den/die ursprünglichen Autor(en) und die Quelle ordnungsgemäß nennen, einen Link zur Creative Commons Lizenz beifügen und angeben, ob Änderungen vorgenommen wurden.

\section{Literatur}

1. Esente P, Giambartolomei A, Gensini GG, Dator C (1983) Coronary reperfusion and Bezold-Jarisch reflex (bradycardia and hypotension). Am J Cardiol 52:221-224. doi:10.1016/0002-9149(83)90111-X

2. Ma SX, Long JP (1991) Central noradrenergic activity is responsible for nitroglycerin-induced cardiovascular effects in the nucleus tractus solitarii. Brain Res 559:297-303

3. Ma SX, Schmid PG, Long JP (1994) Noradrenergic mechanisms and the cardiovascular actions of nitroglycerin. Life Sci 55:1595-1603

4. Shah SP, Waxman S (2013) Two cases of BezoldJarisch reflexinduced by intra-arterial nitroglycerin in critical left main coronary artery stenosis. Tex Heart Inst J 40:484-486

5. Thadani U, Ripley TL (2007) Side effects of using nitrates to treatheartfailureand the acute coronary syndromes, unstable angina and acute myocardial infarction. Expert Opin Drug Saf 6:385-396. doi:10. 1517/14740338.6.4.385

6. Warltier DC, Campagna JA, Carter C (2003) Clinical relevance of the Bezold-Jarisch reflex. Anesthesiology 98:1250-1260 